\title{
The analysis of the impact of environmental factors on turbine performance, develpoment of the models relating the periods between maintenances with selected maintenance factors
}

\author{
Magdalena THIELMANN *1,2 \\ ${ }^{1}$ Łukasiewicz Research Network - Institute of Aviation, Warsaw, Poland \\ ${ }^{2}$ Warsaw Univesity of Technology, Warsaw, Poland
}

\begin{abstract}
There are currently around 18,000 commissioned Gas Turbines in use worldwide, with almost 7,500 long-term service agreements[1]. At the same time, orders for new units increase year by year, and after a decrease in production in 2020 from 353 to 328 new units, from 2022 onwards, the level is planned to rise to the previous level of growth. Gas turbines operate worldwide and are exposed to variations in environmental conditions, such as changes in humidity, temperature, and salinity, which can significantly affect the efficiency and faster degradation of individual components. Based on the unit's maintenance report, there are more than 1,940 event alerts annually. A need exists to create a more dynamic analytical and numerical model that determines the impact of environmental variables on gas turbine stability. It is necessary to analyze and improve existing reliability models, which vary due to configurations and the impact of working conditions. The first step should be an analysis of the impact of environmental factors on turbine performance. This paper describes how the maintenance and inspection model developed from an average value over time model to a model tracking the actual degradation of gas turbines. It includes a comparison ofthree models used in the research, considering the developed methodology for selecting input parameters, their correlation, and their appropriateness for use in further analyses.
\end{abstract}

Keywords: Gas Turbine (GT), numerical model, status parameters, maintenance factors

\section{Introduction}

Considering the analysis of gas turbines, we should consider the different configurations and the mode of operation. The operating mode that we can call Duty Cycle can be defined, i.e., as standby, cyclic, continuous, base load, Peaker, or a backup unit, and it significantly affects the dedicated maintenance period. The turbine inspection can be complete or only cover selected sections of the gas turbine, such as the Hot Gas Path section or Combustion. The period between inspections may be between 10,000 and up to 40,000 hours, but each manufacturer may define particular periods between inspections depending on the purpose of the unit concerned. In addition, if the plant has a long-term service maintenance agreement signed, the equipment work is monitored, and if the permissible range of deviations from the typical nature of the turbine's operating characteristics is exceeded, the alert may occur. It may signal a warning or actual event, and based on preliminary data analysis may be necessary to shut it down for further testing.

Based on experience in maintaining the reliability of gas turbines over the years, guidelines have been developed to determine operating parameters and their impact on the service life of major components such as the combustion chamber, the rotor, or the hot gas path and other critical components. Each manufacturer sets guidelines for servicing and use already at the design phase and subsequent production stage. However, due to the variety of solutions used, the end factors are general, and their scope must be adapted to a larger population. To properly analyze the material degradation, there was a need to get acquainted with these guidelines more closely and expand them with additional thermodynamic and environmental parameters. Many inconsistencies were encountered during the implementation of the first version of the model (Basic Model), which resulted in significant discrepancies in the gas turbine sample

\footnotetext{
${ }^{*}$ Corresponding author: E-mail address: Magdalena.Thielmann.dokt@pw.edu.pl (Magdalena THIELMANN)
} 
results. It was necessary to find the relationships between noticed inconsistencies and optimize the input data. The result of all analyses was the model that includes validating input parameters by grouping them and determining the relationship between the parameters of different groups. Additionally, a weighted quality flag was developed and affected the legitimacy of a given parameter. As a result, we find the coefficient values determining the working conditions and wear of the individual components in the form of a time-changing distribution rather than an averaged value over a given period.

Subsequent analyses have shown that if correlations between different parameter groups and within those groups are applied, one can achieve higher compatibility of the predicted distribution of actual wear of the turbine components and critical parts.

While the analyses can show similarities between the behavior of the subjects and their configuration and working conditions, it is vital in further research to create a learning sample as a base to create the models based on machine learning algorithms, to be able to find similar behaviors at the level of direct measurements to avoid re-failures or forced outages.

The purpose of the research is to define a universal model that designates a maintenance factor that ultimately affects extending or reducing the period between inspections. These studies are the fact that we may avoid fatal failures by considering the impact of environmental factors and the duty cycle of the Gas turbine.

\section{Maintenance factor models}

Primary Maintenance Factors could be defined in different ways and base on various factors. This chapter introduces us to the three models: basic, advanced, and advanced, with correlations between status and load parameters. Advanced models extend the basic model by additional input parameters and correlations between them, and to understand the differences, the examples were added at the end of the definition.

\subsection{Basic model}

All analyses $[2,4-8,11]$ are based on the defined Maintenance Factor (Eq. 1), but the different ways in which individual co-factors are defined and their dependability are the basis for further consideration of the gas turbine consumption curve.

$$
F=\frac{\sum(A \cdot B \cdot C \cdot D \cdot E \cdot X)}{\sum X}
$$

Where:

A - Load Severity Factor $(\mathrm{A} \epsilon 1,1.2)$

- Depends on the percentage of turbine load,

- For partial or nominal load, the coefficient equals 1 ,

- For peak load, the factor equals 1.2,

B - Fuel Severity Factor (B $\epsilon 1,1.5,2.5,3.5,10$ )

- Dependent on the type of fuel used, the value decreases as the fuel's hydrogen content increases. In simple terms, the following shall apply:

$$
\begin{aligned}
& \mathrm{B}=1 \text { for natural gas } \\
& \mathrm{B}=1.5 \text { for refined fuels } \\
& \mathrm{B}=2.5 \text { for crude oil } \\
& \mathrm{B}=3.5 \text { for fuel oil }
\end{aligned}
$$

- In the operating mode specified by the manufacturer, the B factor can be set to 10

C - Injection Severity Factor $(\mathrm{C} \geq 1)$

- Depends on: 
- percentage of steam/water content in the air at the compressor inlet

o the amount of water injected to the amount of fuel injected

D - Trip Severity Factor $(\mathrm{D} \geq 1)$

- It depends on the load on the object at the time of the failure

○ No failure $\mathrm{D}=1$

$\circ \mathrm{D}=\mathrm{d}_{1}+e^{d_{2}}$ \% load , where $\mathrm{d}_{1}$ and $\mathrm{d}_{2}$ are the parameters specified by the manufacturer

E - Start-up Factor

- It depends on the number of hours since the last shutdown to determine whether it was a cold, warm, or hot start.

- For cases where the rotor is not analyzed, the factor is omitted.

$\mathrm{X}$ - number of hours or cycles.

The Maintenance Factor takes values from 1 to 12. The manufacturer may recommend a different review or action scope depending on the Maintenance Factor value received since the last review. In the case of stable operation of the turbine, where other parameters such as temperature, vibration, leakage of the valves do not exceed the permissible range, it is assumed that in the case of:

- F $\epsilon[1,1.5]$ - standard service activities are recommended within a predetermined inspection date,

- F $\epsilon[1.5,5]$ - standard service is recommended before the predetermined inspection date,

- F $\epsilon[5,12]$ - it is recommended to increase the range of service activities with a significantly accelerated period between inspections, which directly depends on the value of factor $\mathrm{F}$, e.g., for factor $\mathrm{F}=12$ and the suggested $\mathrm{X}$ number of hours, the period between inspections suggested by the manufacturer is $\mathrm{X} / 12$ and not $\mathrm{X}$.

The commonly used method for determining how the vital gas turbine's parts wearout, the coefficients of gas turbines was to determine the value averaged over a more extended period, like a year or a month. Its basic dependencies are represented by the diagram (Fig. 1). The elements' expected wear could be determined by referencing the average coefficients determined for the facilities' accumulated performance. Sometimes averaged values are not taken into account, and, instead, the maximum value of individual coefficients in individual cycles is. Supposing the object has worked X1 hours with nominal load and X2hours with peak load in a given cycle, the average according to the equation (Eq. (2)) [9]

$$
\frac{X_{1} \cdot 1+X_{2} \cdot 1.2}{X_{1}+X_{2}}
$$

can be used, or in practice, one could use the maximum parameter for this cycle, the simplified Maintenance Factor that equals 1.2. This approach makes the planned inspections and determination of the device's suitability for further use subject to, e.g., overstatement of parameters in the case of turbines with variable operating parameters and, in fact, a reduction in the time between repairs.

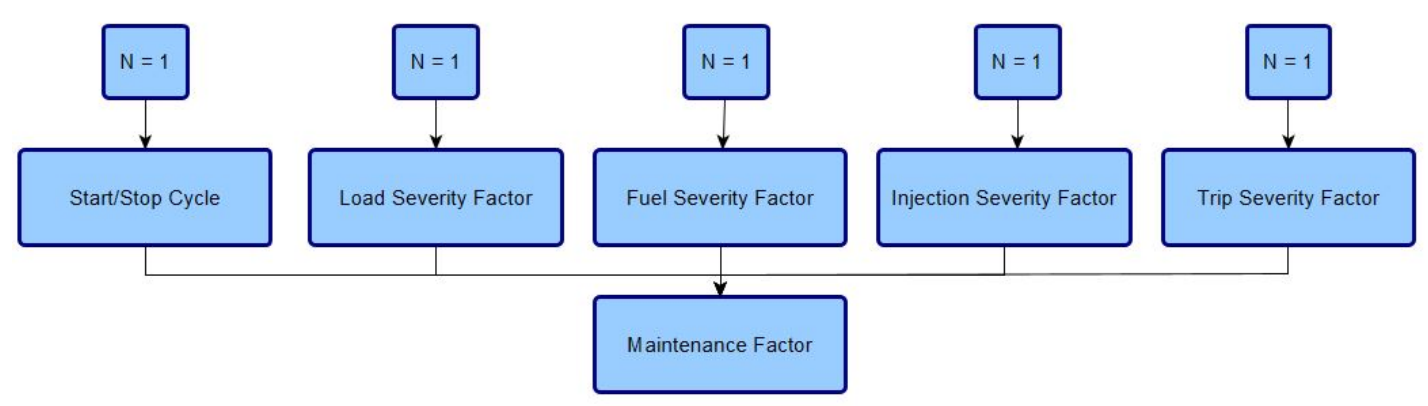

Figure 1. The diagram shows the relationship between input parameters and output factors, where $\mathrm{N}$ is the number of input parameters. 
The diagram (Fig. 1) introduces N, the number of parameters affecting a given factor's value. For example, at the level of the "Start/Stop Cycle" parameter group, it should be noted that $\mathrm{N}=1$ means that only one parameter was taken to determine this factor's value: a state parameter indicating the turbine was switched on. Then, let us analyze the ratio of time when the unit was running during the period considered, e.g.,one year to the length of that period as the percentage of running time. Note that this solution results in an error resulting from averaging parameters already at the input level and each parameter being treated separately.

\subsection{Advanced Model}

The base model analysis showed that input parameters could be obtained as a direct value, but further analysis of the status parameters demonstrated the possibility of using intermediate parameters to provide preliminary validation of input parameters to the model. At this stage, additional parameters were selected that could be grouped into six input groups shown in Fig. 2. Following that, the input parameter was validated based on $\mathrm{N}$ direct or indirect values inside the six groups. The result was the determination of coefficients according to the scheme (Fig. 2) in the minor available time intervals that were aggregated. The validation of the extended model made it possible to determine the actual consumption curve of the gas turbine rather than the averaged ranges, resulting in more accurate reliability analyses as a basis for the decision to extend or shorten the service interval. Studies have shown $[1,3,10]$ that modifying the base model is correct and that the researched numerical model should be developed. The diagram (Fig. 2) notes that compared to the base model, the extended model $\mathrm{N}$ values take from 1.

\subsection{Example}

$\mathrm{N}=5$ for the "Start/Stop Cycle" parameter group. In addition to the previously included state parameters as:

- Turbine switching determines whether the unit's status is running, in acceleration mode, or shut down.

- The speed - the level speed may define the phase of the running unit.

- Flame detection - determines a unit is working. It may be used as a backup parameter when others are not detected.

- Load - may be defined as control response from the unit algorithms or the ratio of generated power to designed output power.

- Generated power - the level or percentage of output power.

- Start/Stop Cycle could be defined between all Starts (shutdown status converts into running status) and Stop (running status converts into shutdown status).

A significant change in the extended model's requirements is no longer accepting the simplification of adopting the entire cycle's maximum parameter value. A new parameter was introduced in further studies to indicate partial load operation, i.e., $\mathrm{A}_{1}<1$. The value of this parameter is further analyzed and currently ranges between 0.6 and 1 . If the object under consideration operates $\mathrm{X}_{0}$ hours under partial load, $\mathrm{X}_{1}$ hours under nominal load, and $\mathrm{X}_{2}$ hoursunder peak load in a given cycle, then the "Start/Stop Cycle" parameter is determined from the following equation

$$
\frac{X_{0} \cdot A_{1}+X_{1} \cdot 1+X_{2} \cdot 1.2}{X_{0}+X_{1}+X_{2}}
$$

\subsection{Advanced Model with the correlation between status and load parameters}

At the next stage of model modification, new parameters were added and assigned into subgroups for each co-factor. The correlations between all subgroups were defined at two levels: input parameters and co-factors.

At first, all input data was split into cycles, then each cycle into the smallest available resolution. This method improves the convergence for the predicted distribution of actual consumption by analyzing the coefficient values of each data point. Previously the co-factor was defined per cycle as a maximum value, but now the cycle is aggregated by sub-cycles with the same operational state. The diagram (Fig. 3) shows the relationship between parameters at each stage of model validation. To better understand the correlation between input parameters and co-factor values, one should look closely at the turbine speed. The turbine speed is the main parameter for determining the load factor value. However, when determining failure factors, it is also an additional parameter that affects the state parameter's value with operating status, failure in the load function, and whether a valid start has occurred. 


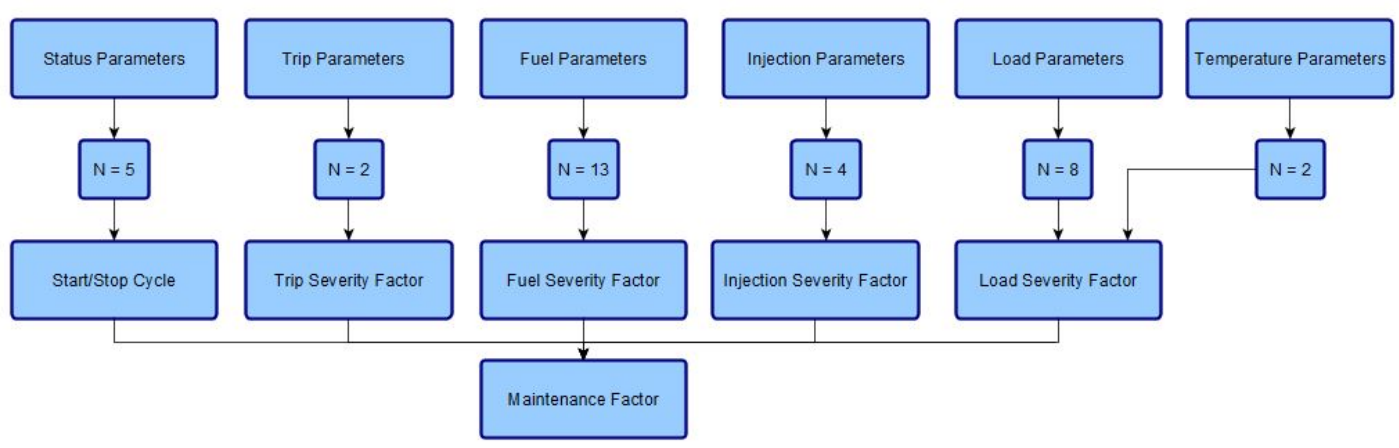

Figure 2. The relationship between input parameters and output factors, where $\mathrm{N}$ is the number of input parameters in a given group.

\section{Example:}

Let's analyze one of the input parameters, turbine speed (TNH, expressed in percent of full speed).

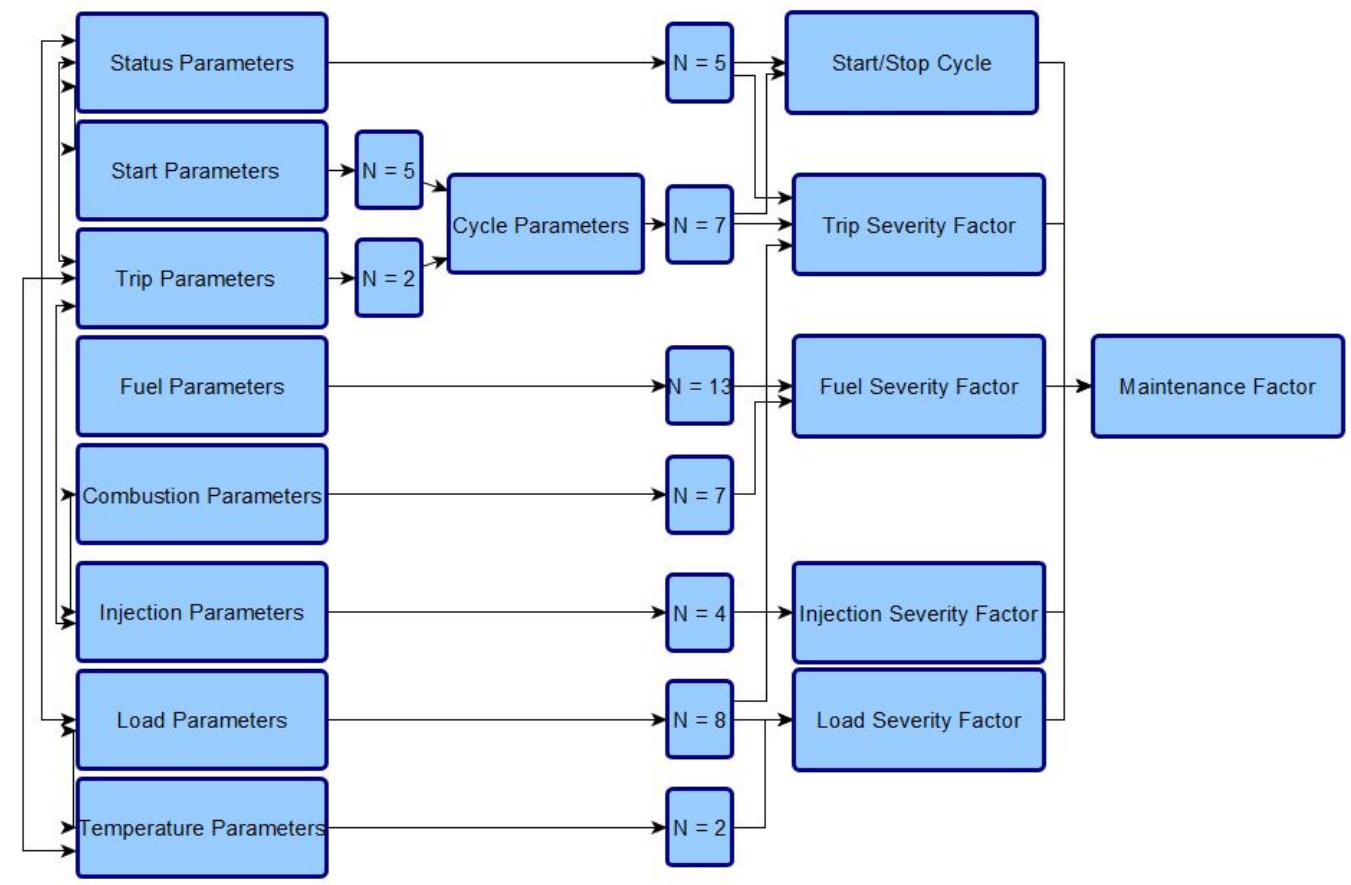

Figure 3. The relationship between the input parameters and the output factor, together with the correlation between the different input parameters, where $\mathrm{N}$ is the number of input parameters in each group

If we have input subgroups such as Status, Start, Trip, Fuel, Combustion, Injection, Load, and Temperature Parameters (Fig. 3), we could closely examine the impact of one that input on most Parameters subgroups.

- Status Parameters: TNH is an additional parameter, strongly correlated, defining a gas turbine's state when direct sensors could not be provided. Based on the trend of TNH change over time,it is possible to define the working stage of equipment: start-up, running, and shutdown.

- Start Parameters: TNH is an additional parameter defining a gas turbine's state when direct state sensors are unavailable. During start-up, with the smallest possible resolution, considering the optimization of the process, it is possible to detect the start based on gas turbine manufacturer guidelines.

- Trip Parameters: TNH is a parallel parameter strongly correlated with the gas turbine's alarm detection sensors. It is crucial to determine the level of TNH when Failure occurred (Trip from load dependency). 
- Fuel Parameters: TNH is not included in these sub-groups of input parameters.

- Combustion Parameters: TNH is not included in these sub-groups of input parameters.

- Injection Parameters: TNH is not included in these sub-groups of input parameters.

- Load Parameters: TNH is the main parameter defining Load Factors, and its values directly impact the cycles aggregated by sub-cycles with the same operational state mentioned previously.

- Temperature Parameters: TNH is an additional parameter used to the Firing Temperature definition by analyzing the relation between values and operational cycle, heat recovery vs. simple cycle.

For further research, it is vital to determine the relationship between input parameters and their contribution to the variability of values of the individual coefficients. The advanced numericalmodel with the correlation between status and load parameters shows dependencies in the correlation matrix (Fig. 4) between the input parameters' relevant subgroups. The correlation matrix (Fig. 4) should be understood as meaning that the input data, direct or indirect, impact coefficients' groups differently as:

- strongly correlated - Input from groups:

- Status,

- Temperature,

- Load.

- poorly correlated - Inputs from groups:

- Fuel,

- Cycle.

The higher the correlation coefficient (the scale on the right to Fig. 4), the more dependent the variables are. The gas turbine operating parameters are necessary to determine the facility's actual operating load, and the second parameter in terms of interdependence is temperature. In contrast, the fuel type affects the remaining parameters only indirectly by influencing temperature. However, note that correlation coefficients only mean the interdependence of the input parameters, not the effect on the final result, which is the F-factor described by equation (Eq.1).

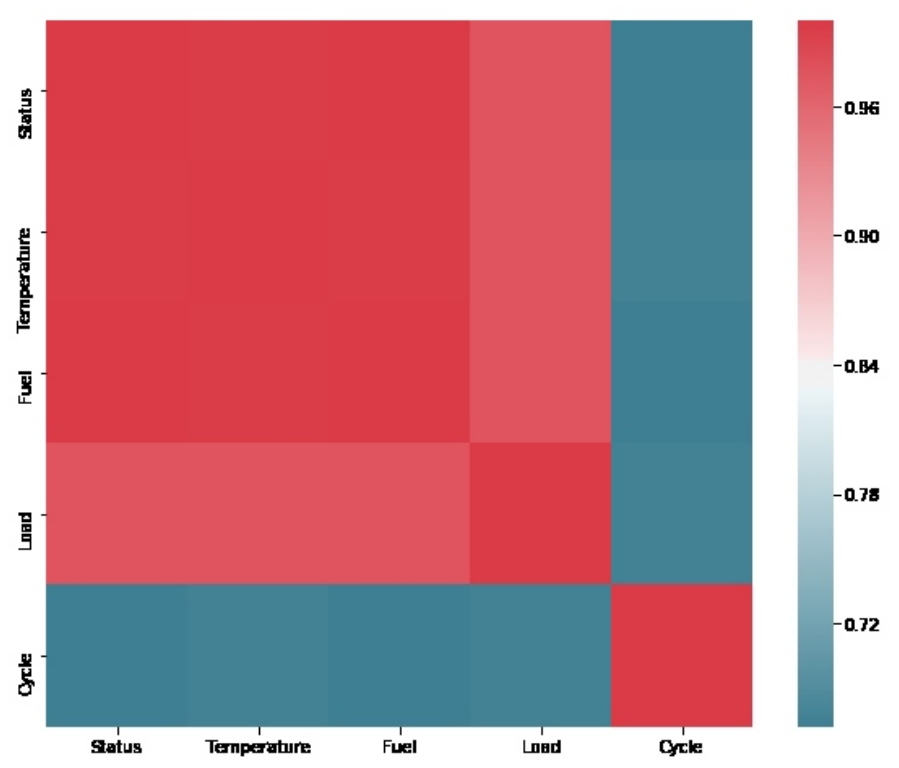

Figure 4. The correlation matrix. A correlation matrix consists of rows and columns showing the variable, displaying the correlation between the variables, and using a linear relationship. The scale from grey to red displays increasing percentage correlation, where red is the highest correlation. i.e., Temperature and Status Parameters are strongly correlated, where Fuel Parameters to Temperature Parameters are less correlated. 


\section{Validation algorithms for the status and load parameters}

An analysis of the different models has shown that it is crucial to introduce the prevalidation of the values of selected gas turbine operating parameters. The relationship between the different values, the ranges of their applicability, and the relationship between them forced the development of a validation model, the outline presented in the diagram (Fig. 5).

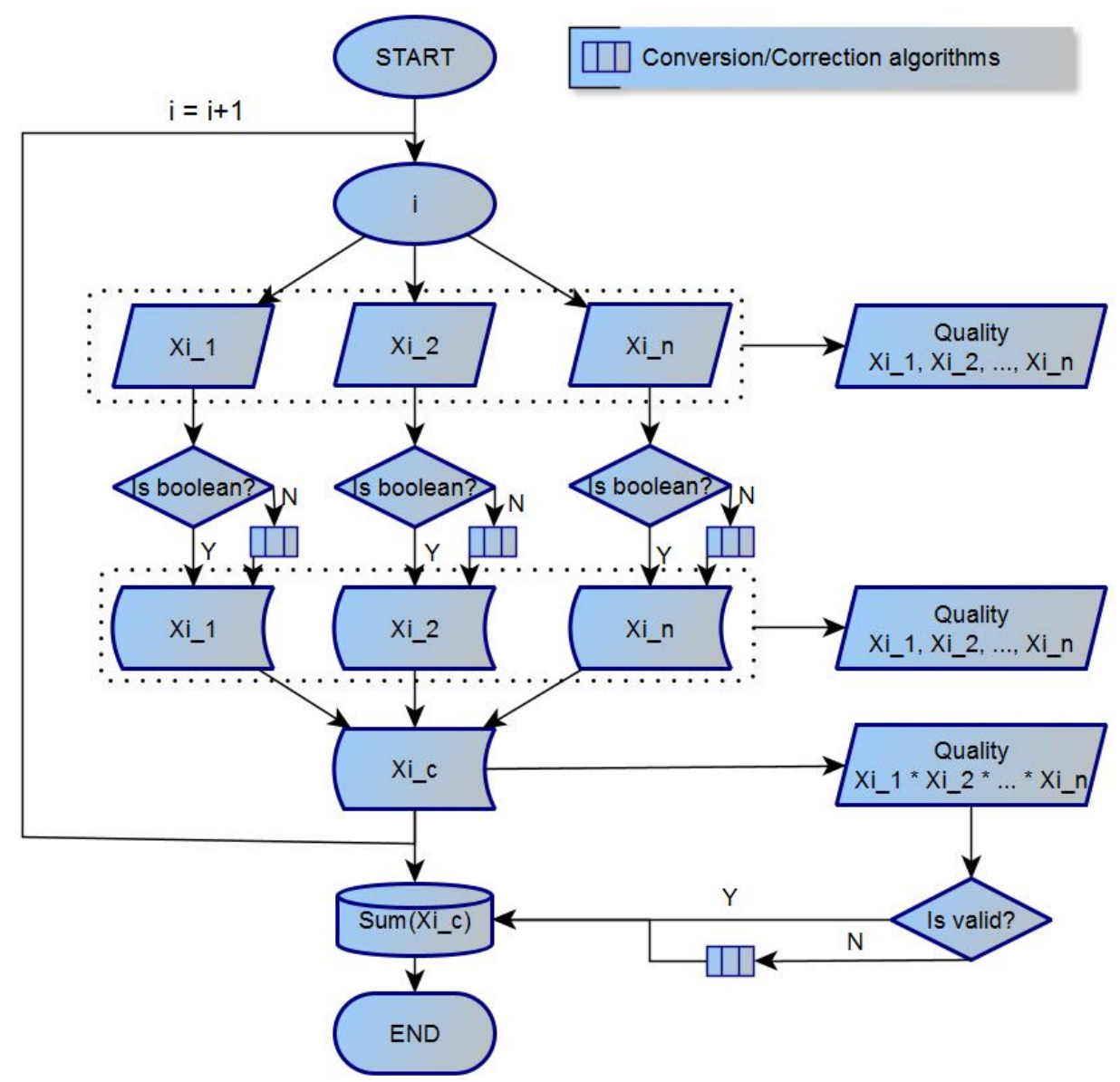

Figure 5. The methodology of input data validation that assigns quality flags to the iterated measure.Conversion/correction process for missing or values with low quality check correlated parameters and fill the gaps

Stage 1:

1. Validating data

2. Designating quality flags for a given point

Stage 2:

3. Providing the data type

4. Defining the correlation between parameters

5. Normalizing data to logical values

Stage 3:

6. Saving data

7. Providing quality flags for a given point

Stage 4: 
8. Converting $\mathrm{N}$ logical data into one parameter

9. Providing a quality flag for a given point (grouped per parameter)

Stage 5:

10. Depending on the value of the designated qualitative flag, the parameter is validated for further analysis

11. If the quality flag value is too low, the next iteration will occur

\subsection{Example of the methodology explained above and presented in Fig. 5}

In order to bring the algorithm of data preparation for further analysis closer, the algorithm for the initial assignment of quality flags is shown (Tab. 1). The following steps are to check the data for a particular input and determine the final value for a given coefficient based on the dependents between them.

Table 1. Level 1 of Figure 5 For i record, apply the first stage of Quality Flags

\begin{tabular}{|c|c|c|c|}
\hline Is unit running & Is unit shutdown & Turbine switching output & Quality [\%] \\
\hline False & True & 0 & 100 \\
\hline False & False & None & 0 \\
\hline True & None & 1 & 50 \\
\hline True & False & 1 & 100 \\
\hline
\end{tabular}

Table 2. Following levels to Table 1 to provide Status Parameters at i row with quality flag.

\begin{tabular}{|c|c|c|c|c|c|c|}
\hline Stage & $\begin{array}{l}\text { Turbine } \\
\text { switching }\end{array}$ & The speed & $\begin{array}{c}\text { Flame } \\
\text { detection }\end{array}$ & Load & $\begin{array}{c}\text { Generated } \\
\text { power }\end{array}$ & Quality \\
\hline \multirow[t]{4}{*}{ Level 1} & 0 & 0 & 0 & $0 \%$ & 0 & 1.0 \\
\hline & None & 40 & 1 & $20 \%$ & 20 & 0.8 \\
\hline & 1 & None & 1 & $80 \%$ & 80 & 0.9 \\
\hline & 1 & 90 & 1 & $80 \%$ & None & 1.0 \\
\hline \multirow[t]{5}{*}{ Level 2} & $\begin{array}{c}\text { Fill gaps } \\
\text { based on } \\
\text { other values }\end{array}$ & $\begin{array}{c}\text { Fill gaps } \\
\text { based on other } \\
\text { parameters or } \\
\text { previous and } \\
\text { following values }\end{array}$ & $\begin{array}{c}\text { Fill gaps } \\
\text { based on } \\
\text { other values }\end{array}$ & & $\begin{array}{c}\text { Fill gaps based } \\
\text { on other } \\
\text { parameters } \\
\text { or previous } \\
\text { and following } \\
\text { values }\end{array}$ & $\begin{array}{c}\text { Decrease } \\
\text { quality }\end{array}$ \\
\hline & 0 & 0 & 0 & $0 \%$ & 0 & 1.0 \\
\hline & 1 & 40 & 1 & $20 \%$ & 20 & 0.64 \\
\hline & 1 & 90 & 1 & $80 \%$ & 80 & 0.72 \\
\hline & 1 & 90 & 1 & $80 \%$ & 80 & 0.8 \\
\hline \multirow[t]{4}{*}{ Level 3} & \multicolumn{5}{|c|}{0} & \\
\hline & \multicolumn{5}{|c|}{1} & \\
\hline & \multicolumn{5}{|c|}{1} & $1.0 \cdot 0.64 \cdot 0.072 \cdot 0.8$ \\
\hline & \multicolumn{5}{|c|}{1} & \\
\hline
\end{tabular}


Table 2. presents the next steps discussed earlier (2nd), and the example concerns Status Parameters at $\mathrm{N}=5$ (Turbine switching, speed, Flame detection, Load, Generated power).

This example should bringthe methodology of how the record for each parameter could be fixed closer with assigned quality flags is presented in Fig. 5. In Level 2 in Tab 2. bold changes are the results of the Conversion/Correction process marked in Fig. 5.

\section{Comparisonof models}

Numerical models of the shortly described three models have shownthat choosing a timestep of the input sample is vital to determine how the primary turbine performance changes over time. The right step size should optimize the received results.If the Maintenance Factor F (Tab.3) is simplified to the stable turbine operation, that depends only on the operating load, and the remaining parameters equal one. The estimated value can be compared using the previously discussed models for a single turbine duty cycle. It can be observed that the impact of the actual operating schedule of the turbine influences the planning of further service work. It is assumed that $\mathrm{F}$ (Eq. 1) values to pre-enter the factor under partial load $<1$, which allows $\mathrm{F}<1$, close to unity while considering the correlation between the input parameters. The A value and dependency under partial load need deeper analysis before defining the Maintenance Factor's impact.

Table 3. Approximate Maintenance Factor F values with percentage reference to the simplified basic model.

\begin{tabular}{|c|c|c|c|c|}
\hline Model & Cycle A & $\Delta$ A & Cycle B & $\Delta$ B \\
\hline Basic Model (Simplified) & 1.0 & - & 1.2 & - \\
\hline Basic Model & 1.0 & $0 \%$ & 1.046 & $12.8 \%$ \\
\hline Advanced Model & 1.0 & $0 \%$ & 1.014 & $15.5 \%$ \\
\hline Advanced Model with correlation & 0.978 & $2.2 \%$ & 1.024 & $14.6 \%$ \\
\hline
\end{tabular}

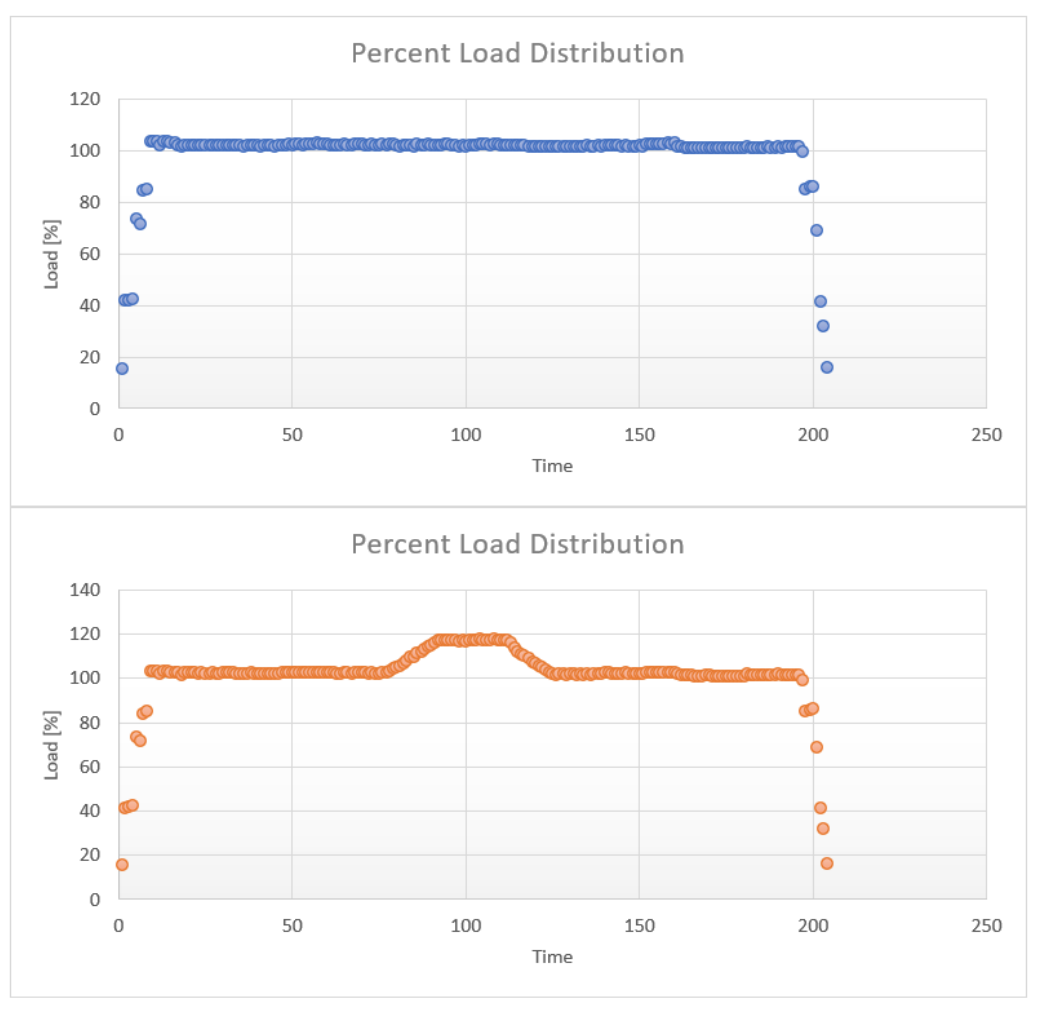

Figure 6. An example of a gas turbine load distribution in one cycle without peak load in cycle A (top graph), with peak load in cycle B (bottom graph). 
The $\Delta \mathrm{A}$ and $\Delta \mathrm{B}$ showed for four models per cycles $\mathrm{A}$ and $\mathrm{B}$ (Tab. 3., Fig.6) provide the percentage of extended hours between service intervals to the Basic Model (simplified) results on the site. It means that Basic and Advanced Models for Cycle A will not change provided by Basic Model (simplified) interval between inspections; however, this interval will be increased by $2.2 \%$. i.e., if the interval hours between inspections from the Basic Model equals 1,000 hours, then from Advanced Model with correlation, this period should be extended to 1022 hours. Note that the value of this difference for the advanced model with correlation is lower than for the advanced model. The results of the analysis show that the interdependence of input parameters for the time interval resulting from the $0.9 \%$ difference $(15.5 \%-14.6 \%)$ suggest that the gas turbine should be switched off prematurely or allowed continued work with a reduced load as well as suggesting close observation of individual alarms. During this period, the risk of failure increases.

\section{Conclusion}

Comparison of the results from numerical models for the shortly described three models has shown that the best accuracy and course of the wear curve based on the coefficients determined requires multilayer validation of the parameters to determine the correlation between the analyzed parameters. The use of the developed algorithm has improved the compatibility of the predicted distribution to actual consumption. The innovation of the solution consists, among other things, in the approach to determining the actual work cycle of the object, that is, the Start-Stop cycle by breaking down a given cycle into sub-cycles of all determined coefficients at individual time points, which required the algorithm shown in the diagram (Fig. 3) with the relationship between the coefficients, rather than treating them separately. The results show that the chosen path is worth further analysis.

\section{Acknowledgments}

The research carried out is part of the Industrial Doctorate Program in the agreement between 3 parties. The Ministry of Science and Higher Education, Warsaw University of Technology, and the Łukasiewicz Research Network - Institute of Aviation.

\section{References}

1. Anping, W. et al. Prognostics of gas turbine: A condition-based maintenance approach based on multi-environmental time similarity. Mechanical Systems, and Signal Processing 109 (2018).

2. Bazazzadeh, M. \& Badihi H.and Shahriari, A. Gas Turbine Engine Control Design Using Fuzzy Logic and Neural Networks. International Journal of Aerospace Engineering 2011.

3. Gul, M. et al. Multi-objective-optimization of process parameters of industrial-gas-turbine fueled with natural gas by using Grey-Taguchi and ANN methods for better performance. Energy Reports 6 (2020).

4. Huadong, M., S., G. \& Xie., M. Performance-based maintenance of gas turbines for reliable control of degraded power systems. Mechanical Systems and Signal Processing 103 (2018).

5. Jasnawitz, J., Masso, J. \& Childs, C. Heavy-Duty Gas Turbine Operating and Maintenance Considerations, (GE Gas Turbine Reference Library).

6. Kejian, W. et al. Heavy-duty gas turbine performance state monitoring method based on generalized regression neural network and boxplot analysis. Patent 2019.

7. Meherwan, P. B. Gas Turbine Engineering Handbook Fourth Edition (Butterworth-Heinemann, 2012).

8. Mohammadi, R., Naderi, E., Khorasani, K. \& Hashtrudi-Zad, S. Fault diagnosis of gas turbine engines by using dynamic neural networks in booktitle IEEE 54th International Midwest Symposium on Circuits and Systems (MWSCAS) (2011), 1-4.

9. Slade, S. \& Palmer, C. Worldwide Gas Turbine Market Report,Turbomachinery Magazine,Handbook (2021).

10. Tahan, M., Tsoutsanis, E., Muhammad, M. \& Karim, A. Performance-based health monitoring, diagnostics and prognostics for condition-based maintenance of gas turbines: A review. Applied Energy 198 (2017).

11. Xusheng, Y., Mingliang B.and Jinfu, L., L., J. \& Daren, Y. Gas path fault diagnosis for gas turbine group based on deep transfer learning. Measurement 181 (2021). 